\title{
Superconductivity in the presence of a pseudogap induced by local charge density waves
}

\author{
R. SZCZEŚSNIAK ${ }^{1}$, D. SZCZECŚNIAK ${ }^{2,3 *}$ \\ ${ }^{1}$ Institute of Physics, Częstochowa University of Technology, \\ Armii Krajowej Ave. 19, 42-200 Częstochowa, Poland \\ ${ }^{2}$ Institute of Physics, Jan Dtugosz University in Czestochowa, \\ Armii Krajowej Ave. 13/15, 42-200 Częstochowa, Poland \\ ${ }^{3}$ Condensed Matter Physics Laboratory UMR 6087, University of Maine, \\ Olivier Messiaen Ave., 72-085 Le Mans, France \\ * Corresponding author.E-mail:d.szczesniak@ajd.czest.pl
}

Received June 24, 2010; accepted May 18, 2011; available on-line November 8, 2011

A model describing the thermodynamic properties of a superconducting phase in the presence of a pseudogap induced by local charge density waves is presented in the paper. Within the framework of the model calculations it is shown that the obtained phase diagram reproduces properly, in a qualitative way, the phase diagram of the superconducting state for $\mathrm{BaPb}_{1-x} \mathrm{Bi}_{\mathrm{x}} \mathrm{O}_{3}$. It is further shown that the pseudogap very strongly reduces the value of the order parameter for the superconducting phase, whereas the value of the electron effective mass and the energy shift function are independent of the pseudogap.

\section{Superconductors / Thermodynamic properties}

\section{Introduction}

The $\mathrm{BaPb}_{1-x} \mathrm{Bi}_{x} \mathrm{O}_{3}$ (BPBO) superconductor was for the first time synthesized by Sleight et al. in 1975 [1]. Due to the fact that the superconducting phase in $\mathrm{BPBO}$ is induced by electron-phonon interaction, the thermodynamic properties of the described compound were in the literature tentatively explained within the framework of the classical Eliashberg formalism [2-5] (for a discussion of the Eliashberg equations, originally formulated by G.M. Eliashberg [5], we refer to [6]). In our opinion, there are strong arguments against the correctness of such a procedure. First of all, the classical Eliashberg theory predicts a completely different phase diagram for the superconducting phase than the one observed for the BPBO superconductor. The phase diagram predicted by the Eliashberg theory and the diagram observed for BPBO $[9,10]$ are presented schematically in Fig. 1. It is easy to spot the great qualitative differences between these diagrams. From the considerations made within the framework of the classical Eliashberg theory it is inferred that the superconducting phase exists in the whole range of the $x$ parameter and the critical temperature takes the highest value for the half-filled electronic band. However, in the phase diagram for BPBO it can be clearly seen that the maximum value of the critical temperature is observed for $x \cong 0.3\left(T_{\mathrm{c} \max } \cong 13 \mathrm{~K}\right)$ and then the superconducting phase quickly disappears. Secondly, the classical Eliashberg theory describes a physical system that can exist in the metallic state or in the superconducting state. The phase diagram obtained for BPBO proves, however, that this compound can exist in three distinct states: metallic, superconducting and semiconducting [10].

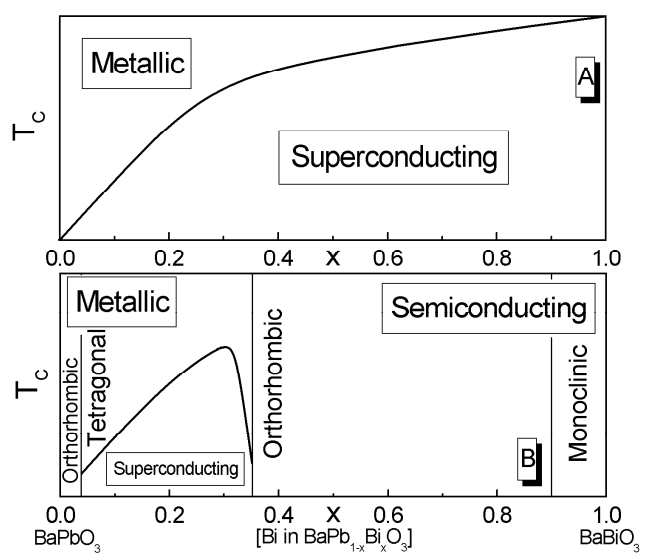

Fig. 1 (A) Phase diagram predicted by the classical Eliashberg theory [9]. (B) Experimentally determined phase diagram for BPBO [10]. In both figures $T_{\mathrm{c}}$ denotes the critical temperature; $x \sim\langle n\rangle$ was assumed, where $\langle n\rangle$ is the average number of electrons at the node. 
The complicated structure of the phase diagram for $\mathrm{BPBO}$ can be understood based on the results obtained for pure $\mathrm{BaBiO}_{3}$ (BBO) [11-14], X-ray absorption fine structure (XAFS) measurements [15] and optical reflectivity measurements $[17,19]$. It has been shown experimentally that the BBO compound is not a good metal with a half-filled electronic band, but it is in the semiconducting state. The reason for this is directly connected with the existence of static charge density waves in BBO, which are induced by a Peierls' instability [20]. The XAFS measurements have additionally proven that, with decreasing $\mathrm{Bi}$ content and increasing $\mathrm{Pb}$ content, the charge density waves are the subject of constant weakening, which means that doping by lead destroys the long-range order CDW in BPBO. The peak in the optical conductivity versus frequency, i.e. the optical energy gap, behaves in an analogous way. The optical gap does not vanish, not even at the transition from the superconducting state to the metallic state $(x=0.35)$, but is still observed in the metallic state $(0.2<x<0.35)$, where a pseudogap was detected. The presented experimental results explicitly suggest that the pseudogap in the electron density of states is connected with the existence of local charge density waves, the remains of the static CDW observed in pure BBO.

Taking into consideration the conclusions from the quoted facts, it has to be stated that the properties of the superconducting phase in BPBO should be described within the framework of an essentially modified Eliashberg theory. An adequate model, in which it has been assumed that the superconducting state is induced by electron-phonon interaction and the pseudogap in the electron density of states is connected with the existence of local charge density waves, is proposed in the present paper.

\section{The model}

In the simplest case the coupling between the electron gas and the vibrations of the crystal lattice is described by the Fröhlich hamiltonian [2]:

$$
\begin{aligned}
& H=\sum_{\mathrm{k} \sigma}\left(\varepsilon_{\mathrm{k}}-\mu\right) c_{\mathrm{k} \sigma}^{\dagger} c_{\mathrm{k} \sigma}+\sum_{\mathrm{q}} \omega_{\mathrm{q}} b_{\mathrm{q}}^{\dagger} b_{\mathrm{q}} \\
& +\sum_{\mathrm{kq} \sigma} g_{\mathrm{k}, \mathrm{k}+\mathrm{q}} c_{\mathrm{k}+\mathrm{q} \sigma}^{\dagger} c_{\mathrm{k} \sigma}\left(b_{-\mathrm{q}}^{\dagger}+b_{\mathrm{q}}\right)
\end{aligned}
$$

where $c_{\mathrm{k} \sigma}^{\dagger}$ denotes the electron annihilation (creation) operator in the Bloch state with the momentum $\mathrm{k}$ and $\operatorname{spin} \sigma ; b_{\mathrm{q}}^{\dagger}$ is the phonon annihilation (creation) operator. The function $\varepsilon_{\mathrm{k}}$ defines the electronic band energy, $\mu$ is the chemical potential, and $\omega_{\mathrm{q}}$ determines the phonon energy with the momentum q. The matrix elements that describe the electron-phonon interaction are denoted by the symbol $g_{k, k+q}$.

The electronic band structure is modeled by a threedimensional band with the nearest neighbor hopping integral $t ; \varepsilon_{\mathrm{k}}=-2 t\left[\cos \left(k_{x}\right)+\cos \left(k_{y}\right)+\right.$ $\left.\cos \left(k_{z}\right)\right]$. In the presented model the electron density of states function was approximated by a constant density of states at the Fermi surface $\rho(0)$. In this case $\rho(0)=1 /(2 \mathrm{~W})$, where $W$ is the half band width; $W=6 t$. In the numerical calculations we take $t$ as an energy unit.

In order to bring out the Eliashberg equations, the Fröhlich hamiltonian should be rewritten with the use of the Nambu spinors $\psi_{\mathrm{k}}, \psi_{\mathrm{k}}^{\dagger}[4]$ :

$\psi_{\mathrm{k}} \equiv\left(\begin{array}{c}\mathrm{ck} \uparrow \\ c_{-\mathrm{k} \downarrow}^{\dagger}\end{array}\right), \quad \psi_{\mathrm{k}}^{\dagger} \equiv\left(c_{\mathrm{k} \uparrow}^{\dagger} c_{-\mathrm{k} \downarrow}\right)$

Then:

$$
\begin{aligned}
& H=\sum_{\mathrm{k}}\left(\varepsilon_{\mathrm{k}}-\mu\right) \psi_{\mathrm{k}}^{\dagger} \tau_{3} \psi_{\mathrm{k}}+\sum_{\mathrm{q}} \omega_{\mathrm{q}} b_{\mathrm{q}}^{\dagger} b_{\mathrm{q}} \\
& +\sum_{\mathrm{kq}} \mathrm{g}_{\mathrm{k}, \mathrm{k}+\mathrm{q}} \psi_{\mathrm{k}+\mathrm{q}}^{\dagger} \tau_{3} \psi_{\mathrm{k}}\left(b_{-\mathrm{q}}^{\dagger}+b_{\mathrm{q}}\right)
\end{aligned}
$$

where $\tau_{3}$ is one of the four Pauli matrices: $\tau_{0}-\tau_{3}$.

The new form of the Fröhlich hamiltonian enables us to bring out the Dyson equation, which can be used to calculate the matrix self-energy $\sum_{\mathrm{k}}\left(i \omega_{m}\right)$ :

$\sum_{\mathrm{k}}\left(i \omega_{m}\right)=\mathrm{G}_{0 \mathrm{k}}^{-1}\left(i \omega_{m}\right)-\mathrm{G}_{\mathrm{k}}^{-1}\left(i \omega_{m}\right)$

In Eq. $4 \mathrm{G}_{0 \mathrm{k}}\left(i \omega_{m}\right)$ is the electronic Green function for the gas of the non-interacting electrons:

$$
G_{0 \mathrm{k}}\left(i \omega_{m}\right) \equiv\left(i \omega_{m} \tau_{0}-\left(\varepsilon_{\mathrm{k}}-\mu\right) \tau_{3}\right)^{-1}
$$

whereas $G_{\mathrm{k}}\left(i \omega_{m}\right)$ denotes the full electronic Green function:

$$
G_{\mathrm{k}}\left(i \omega_{m}\right) \equiv\left(\begin{array}{ll}
\left\langle\left\langle\mathrm{c}_{\mathrm{k} \uparrow} \mid \mathrm{c}_{\mathrm{k} \uparrow}^{\dagger}\right\rangle\right\rangle_{i \omega_{m}} & \left\langle\left\langle\mathrm{c}_{\mathrm{k} \uparrow} \mid \mathrm{c}_{-\mathrm{k} \downarrow}\right\rangle\right\rangle_{i \omega_{m}} \\
\left\langle\left\langle\mathrm{c}_{-\mathrm{k} \downarrow}^{\dagger} \mid \mathrm{c}_{\mathrm{k} \uparrow}^{\dagger}\right\rangle\right\rangle_{i \omega_{m}} & \left\langle\left\langle\mathrm{c}_{-\mathrm{k} \downarrow}^{\dagger} \mid \mathrm{c}_{-\mathrm{k} \downarrow}\right\rangle\right\rangle_{i \omega_{m}}
\end{array}\right)
$$

The Matsubara frequencies are defined by the formula $\omega_{m} \equiv(\pi / \beta)(2 m-1)$, where $\beta$ is the inverted temperature; $\beta \equiv\left(\mathrm{k}_{\mathrm{B}} T\right)^{-1}, \mathrm{k}_{\mathrm{B}}$ is the Boltzmann constant.

The pseudogap in the electron density of states was introduced into the Eliashberg formalism by renormalization of the Green function $G_{0 \mathrm{k}}\left(i \omega_{m}\right)$ in the Dyson equation:

$$
G_{0 \mathrm{k}}\left(i \omega_{m}\right) \rightarrow \mathrm{G}_{0 \mathrm{k}}^{\mathrm{PG}}\left(i \omega_{m}\right) \equiv-\frac{i \omega_{m} \tau_{0}+\left(\varepsilon_{\mathrm{k}}-\mu\right) \tau_{3}}{\omega_{m}^{2}+\left(\varepsilon_{\mathrm{k}}-\mu\right)^{2}+[G\langle n\rangle]^{2}}\langle n\rangle-\frac{i \omega_{m} \tau_{0}+\left(\varepsilon_{\mathrm{k}}-\mu\right) \tau_{3}}{\omega_{m}^{2}+\left(\varepsilon_{\mathrm{k}}-\mu\right)^{2}}[1-\langle n\rangle]
$$


In Eq. $7\langle n\rangle$ stands for the average number of electrons at the node; $G$ is the maximum width of the pseudogap. It is easy to notice that the width of the pseudogap for a fixed number of electrons is defined by the product $G\langle n\rangle$. On the other hand, the depth of the pseudogap is defined by the terms $\langle n\rangle$ and $1-\langle n\rangle$ that appear in the numerators in Eq. 7. In the analyzed model, the pseudogap is deepest and widest for the half-filled electronic band. In the case of the BPBO superconductor the procedure of the parametric description of the pseudogap postulated in Eq. 7 can be motivated by the experimental results presented in [10], where it has been shown that the size of the pseudogap increases with increasing average number of electrons at the node. It is also worth noting that the presented model qualitatively reproduces the dependence of the pseudogap on the average number of electrons at the node that was calculated numerically in [22].

The set of Eliashberg equations with the pseudogap was brought out in the usual, selfconsistent way [5-7]. The following results were obtained:

$$
\begin{aligned}
& Z_{l}=1+\frac{1}{\omega_{l} \beta} \sum_{\mathrm{k}} \sum_{m=1}^{M} K^{-}(l, m)\left[\mathrm{Z}_{m}+F_{\mathrm{k}}(m)-1\right] \omega_{m} D_{\mathrm{k}}^{-1}(m) \\
& \chi_{l}=-\frac{1}{\beta} \sum_{\mathrm{k}} \sum_{m=1}^{M} \mathrm{~K}^{+}(l, m)\left[F_{\mathrm{k}}(m)\left(\varepsilon_{\mathrm{k}}-\mu\right)+\chi_{m}\right] D_{\mathrm{k}}^{-1}(m) \\
& \varphi_{l}=\frac{1}{\beta} \sum_{\mathrm{k}} \sum_{m=1}^{M} K^{+}(l, m) \varphi_{m} D_{\mathrm{k}}^{-1}(m) \\
& \langle n\rangle=1-\frac{2}{\beta} \sum_{\mathrm{k}} \sum_{m=-\infty}^{+\infty}\left[F_{\mathrm{k}}(m)\left(\varepsilon_{\mathrm{k}}-\mu\right)+\chi_{m}\right] D_{\mathrm{k}}^{-1}(m)
\end{aligned}
$$

where:

$$
D_{\mathrm{k}}(m) \equiv\left[\omega_{m}\left(Z_{m}+F_{\mathrm{k}}(m)-1\right)\right]^{2}+\left[F_{\mathrm{k}}(m)\left(\varepsilon_{\mathrm{k}}-\mu\right)+\chi_{m}\right]^{2}+\varphi_{m}^{2}
$$

In Eqs. 8-11 $Z_{l} \equiv Z\left(i w_{l}\right)$ denotes the wave function renormalization factor, $\chi_{l} \equiv \chi\left(i w_{l}\right)$ stands for the energy shift function, $\varphi_{l} \equiv \varphi\left(i w_{l}\right)$ for the order parameter function. Let us notice that in the Eliashberg formalism, the order parameter for the superconducting phase $\Delta_{l} \equiv \Delta\left(i w_{l}\right)$ is defined by the ratio $\varphi_{l} / Z_{l}$. The functions $K^{ \pm}(l, m)$ were defined by the following expression:

$$
K^{ \pm}(l, m) \equiv K(l-m) \pm K(l+m-1)
$$

where $K(l-m)$ is the pairing kernel:

$$
K(l-m) \equiv \int_{0}^{+\infty} d \Omega \frac{2 \alpha^{2} F(\Omega) \Omega}{\left(\omega_{l}-\omega_{m}\right)^{2}+\Omega^{2}}
$$

The symbol $\alpha^{2} F(\Omega)$ in Eq. 14 denotes the Eliashberg function. The calculation of the Eliashberg function from first principles for BPBO is a very difficult task. For this reason the pairing kernel $K(l-m)$ was simplified according to the procedure proposed by Kresin [23]:

$K(l-m) \approx \lambda \frac{v^{2}}{(l-m)^{2}+v^{2}}$

where $v \equiv \beta_{\omega_{D}} / 2 \pi, \quad \omega_{D}$ is the Debye phonon frequency and $\lambda$ is the electron-phonon coupling function; $\lambda \equiv 2 \int_{0}^{+\infty} d \Omega \alpha^{2} F(\Omega) / \Omega$. In the numerical calculations $\omega_{D}=1.5 t$ and $\lambda=2 t$ were assumed. It needs to be mentioned that the application of Kresin's approximation causes a presentation of only qualitative results in the paper.

Taking into consideration the presence of a pseudogap leads to the appearance of the $F_{\mathrm{k}}(m)$ function in the Eliashberg equations:

$$
F_{\mathrm{k}}(m) \equiv \frac{\omega_{m}^{2}+\left(\varepsilon_{\mathrm{k}}-\mu\right)^{2}+[G\langle n\rangle]^{2}}{\omega_{m}^{2}+\left(\varepsilon_{\mathrm{k}}-\mu\right)^{2}+[1-\langle n\rangle][G\langle n\rangle]^{2}}
$$

The $F_{\mathrm{k}}(m)$ function has a complicated structure because it depends on the energy value, as well as on the Matsubara frequency. In the proposed model the $F_{\mathrm{k}}(m)$ function will be approximated by its value on the Fermi surface:

$$
F_{\mathrm{k}}(m) \approx F(m) \equiv \frac{\omega_{m}^{2}+[G\langle n\rangle]^{2}}{\omega_{m}^{2}+[1-\langle n\rangle][G\langle n\rangle]^{2}}
$$

Additionally, the numerical analysis of the Eliashberg equations can be significantly simplified when the sums over momenta are replaced by the integrals over energies. The obtained integrals should then be calculated analytically. The details of the procedure are given below: 
$\sum_{\mathrm{k}} D_{\mathrm{k}}^{-1}(m) \approx \rho(0) \int_{-W}^{W} D_{\varepsilon}^{-1}(m) d \varepsilon=A(m)$

and

$\sum_{\mathrm{k}}\left[F(m)\left(\varepsilon_{\mathrm{k}}-\mu\right)+\chi_{m}\right] D_{\mathrm{k}}^{-1}(m) \approx \rho(0) \int_{-W}^{W}\left[F(m)(\varepsilon-\mu)+\chi_{m}\right] D_{\varepsilon}^{-1}(m) d \varepsilon=B(m)$

where

$$
\begin{aligned}
& A(m) \equiv \rho(0) R_{1}\left(\chi_{m}-F(m) \mu, \sqrt{\left[\omega_{m}\left(Z_{m}+F(m)-1\right)\right]^{2}+\varphi_{m}^{2}}, F(m)\right) \\
& B(m) \equiv \rho(0) R_{2}\left(\chi_{m}-F(m) \mu, \sqrt{\left[\omega_{m}\left(Z_{m}+F(m)-1\right)\right]^{2}+\varphi_{m}^{2}}, F(m)\right)
\end{aligned}
$$

The functions $R_{1}$ and $R_{2}$ are defined by the following expressions:

$$
\begin{aligned}
& R_{1}\left(x_{1}, x_{2}, x_{3}\right) \equiv \frac{1}{x_{2} x_{3}}\left[\operatorname{arcTan}\left(\frac{x_{3} W+x_{1}}{x_{2}}\right)+\operatorname{arcTan}\left(\frac{x_{3} W-x_{1}}{x_{2}}\right)\right] \\
& R_{2}\left(x_{1}, x_{2}, x_{3}\right) \equiv \frac{1}{2 x_{3}} \ln \left(\frac{\left(x_{3} W+x_{1}\right)^{2}+x_{2}^{2}}{\left(x_{3} W-x_{1}\right)^{2}+x_{2}^{2}}\right)
\end{aligned}
$$

\section{Numerical results}

\section{A. Influence of the width of the pseudogap on the values of the $\varphi, Z$ and $\chi$ functions}

A numerical analysis of the Eliashberg equations was conducted for four hundred Matsubara frequencies $(M=400) ; k_{B} T=10^{-3} t$ and $\langle n\rangle=0.7$ was assumed.

The dependence of the order parameter function on the Matsubara frequency for the selected values of $G$ is presented in Fig. 2a. It can be noticed that the order parameter function decreases with increasing maximum width of the pseudogap. This fact can be easily explained when remembering that the pseudogap always opens itself on the Fermi surface and the $G\langle n\rangle$ product defines the width of the pseudogap. Thus, when the value of the $G$ parameter increases, the width of the pseudogap increases as well and, as a consequence, the number of electrons that are able to form Cooper pairs near the Fermi surface must decrease. The presented results also prove that the $\varphi_{m}$ function always reaches its maximum for $m=1$, independently of the assumed value of the $G$ parameter.

Figs. $2 \mathrm{~b}$ and $2 \mathrm{c}$ show the dependence of the wave function renormalization factor and energy shift on successive Matsubara frequencies, $G=10^{-4} t$ was assumed. Only one value of the $G$ parameter was selected, because it has been stated that the $Z_{m}$ and $\chi_{m}$ functions do not depend on the width of the pseudogap. In the Eliashberg formalism, the $Z_{m}$ function describes the electron effective mass $\left(m_{e}^{*}\right)$ whereas the $\chi_{m}$ function renormalizes the value of the chemical potential. On the basis of the results presented in Figs. $2 \mathrm{~b}$ and $2 \mathrm{c}$ it has to be concluded that the change of width of the pseudogap in the electron density of states does not have any influence on the electron effective mass value and does not change the function that renormalizes the chemical potential. It is also worth noticing that, like the order parameter function, the $Z_{m}$ and $\chi_{m}$ functions take their extreme values for $m=1$.

\section{B. Dependence of the $\varphi, Z, \chi$ and $\mu$ functions on $\langle n\rangle$}

In the present subchapter the dependence of the solutions of the Eliashberg equations on the average number of electrons at the node is analyzed. The dependence is far more complicated in the investigated model than in the classical Eliashberg theory, due to the fact that both the width and depth of the pseudogap are parameterized by $\langle n\rangle$.

In Fig. $3 a$ the dependence of the $\varphi_{m}$ function on the Matsubara frequency for selected values of $\langle n\rangle$ is presented. Identical values for the maximum width of the pseudogap and temperature, $G=10^{-3} t$ and $\mathrm{k}_{\mathrm{B}} T=10^{-3} t$, were assumed. When analyzing the presented results it was concluded that with an increase of the average number of the electrons at the node, the $\varphi_{m}$ function first increases $(\langle n\rangle=0.2$ and $\langle n\rangle$ $=0.4)$, and then begins to decrease $(\langle n\rangle=0.6$ and $\langle n\rangle=0.8)$. It may be noticed that the behavior of the function $\varphi_{m}$ is totally different from the one predicted by the classical Eliashberg theory $(G=0)$. The dependence of the order parameter function on the average number of electrons at the node within the framework of the classical formalism was analyzed in [9]. It was shown there that the values of the $\varphi_{m}$ function increase with increasing values of the parameter $\langle n\rangle$. The unusual dependence of the $\varphi_{m}$ function on $\langle n\rangle$ is connected with the fact that with increasing values of the $\langle n\rangle$ parameter, the number of electronic states near the Fermi surface initially increases and then begins to decrease, due to the increase of the width and depth of the pseudogap. 

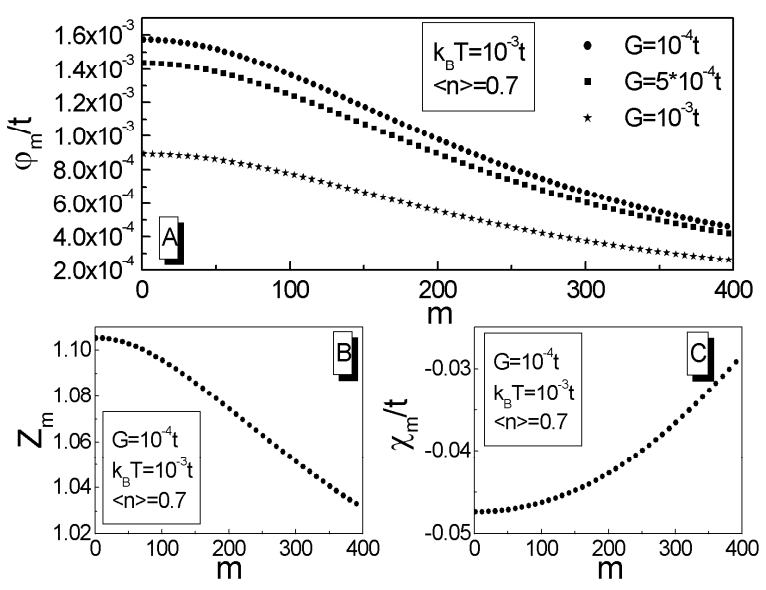

Fig. 2 (A) Dependence of the $\varphi_{\mathrm{m}}$ function on the Matsubara frequency for selected values of the $G$ parameter. (B) Dependence of the $Z_{m}$ function and (C) $\chi_{m}$ function on the Matsubara frequency for $G=10^{-4} t$.
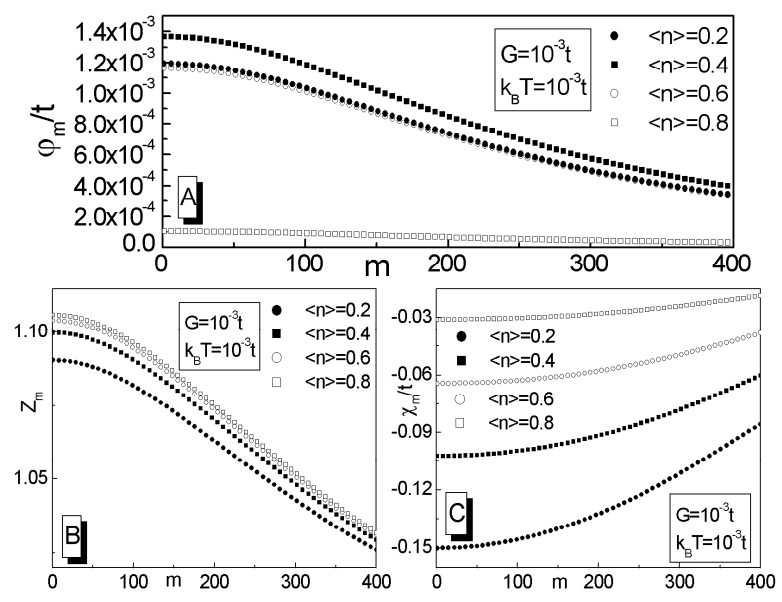

Fig. 3 (A) Dependence of the $\varphi_{m}$ function, (B) $\mathrm{Z}_{\mathrm{m}}$ function and (C) $\chi_{m}$ function on the Matsubara frequency for selected values of the average number of electrons at the node $\langle n\rangle$. $G=10^{-3} t$ and $\mathrm{k}_{\mathrm{B}} T=10^{-3} t$ were assumed.

The wave function renormalization factor and energy shift as a function of the Matsubara frequency for the selected values of the $\langle n\rangle$ parameter are presented in Figs. $3 \mathrm{~b}$ and $3 \mathrm{c}$. As before, $G=10^{-3} t$ and $\mathrm{k}_{\mathrm{B}} T=10^{-3} t$ were assumed. It can be noticed that the values of the wave function renormalization factor and of the energy shift function increase with increasing values of the $\langle n\rangle$ parameter. The presented result proves that the existence of the pseudogap in the electron density of states does not change the dependence of the $Z_{m}$ and $\chi_{m}$ functions on $\langle n\rangle$, when compared to the results obtained when the analysis is based on the classical Eliashberg equations [9].
The dependence of the order parameter, wave function renormalization factor, and the energy shift on the average number of electrons at the node can be fully investigated by analyzing the behavior of those functions for the first Matsubara frequency.

The most relevant result achieved in the work is presented in Fig. 4; it is the dependence of the $\varphi_{\mathrm{m}=1}$ function on $\langle n\rangle$ for selected values of the $G$ parameter. On the basis of the presented results, it has been stated that, with the increase of the $G$ parameter, the superconducting phase vanishes from the side of the high values of $\langle n\rangle$. In particular, for the case in which the maximum width of the pseudogap is equal to two and a half-fold value of the considered temperature, the superconducting phase appears in the range in which the superconducting state for BPBO is observed.

Figs. 5a and 5b show plots of $Z_{\mathrm{m}=1}$ and $\chi_{\mathrm{m}=1}$ versus $\langle n\rangle$. Additionally, Fig. 5b includes an insert, in which the dependence of the chemical potential on the average number of electrons at the node is plotted (the way, how the potential was calculated, is precisely described in Appendix A).

It can be noticed that the wave function renormalization factor, the energy shift function and the chemical potential reach their maximum values for the half-filled electronic band. It needs to be underlined that the presented courses of the $Z_{m=1}, \chi_{m=1}$ and $\mu$ functions do not differ from the courses of those functions for the case of $G=0$ [9].

\section{Temperature dependence of the order parameter}

The dependence of the order parameter $\Delta$ on the temperature, for the case when the influence of the pseudogap on the superconducting state is relatively small $\left(G=10^{-4} t,\langle n\rangle=1\right)$ and for the opposite case $\left(G=5 \times 10^{-3} t,\langle n\rangle=0.175\right)$, is described in this subchapter.

Figs. $6 \mathrm{a}$ and $6 \mathrm{~b}$ show the order parameter as a function of the Matsubara frequency for selected values of temperature in the two cases. It is worth pointing out that for the small pseudogap the critical temperature is equal to $\mathrm{k}_{\mathrm{B}} T_{\mathrm{c}}=117.09 \times 10^{-5} t$, whereas for the large pseudogap $\mathrm{k}_{\mathrm{B}} T_{\mathrm{c}}=1053.36 \times 10^{-6} t$.

Within the framework of the Eliashberg formalism the strict form of the $\Delta(T)$ function is determined by the following equation:

$\Delta(T)=\operatorname{Re}[\Delta(\omega=\Delta(T), T)]$

where the symbol $\operatorname{Re}[\Delta(\omega)]$ denotes the real part of the order parameter function on the real axis. The function $\Delta(\omega)$ is determined from the analytical development of the function $\Delta_{m}$ [25] and can be described by the following formula:

$$
\Delta(\omega)=\frac{p_{\Delta 1}+p_{\Delta 2} \omega+\ldots+p_{\Delta r} \omega^{r-1}}{q_{\Delta 1}+q_{\Delta 2} \omega+\ldots+q_{\Delta r} \omega^{r-1}+\omega^{r}}
$$

where $\mathrm{p}_{\Delta n}$ and $\mathrm{q}_{\Delta n}$ are numerical coefficients; $r$ is equal to 200 . 


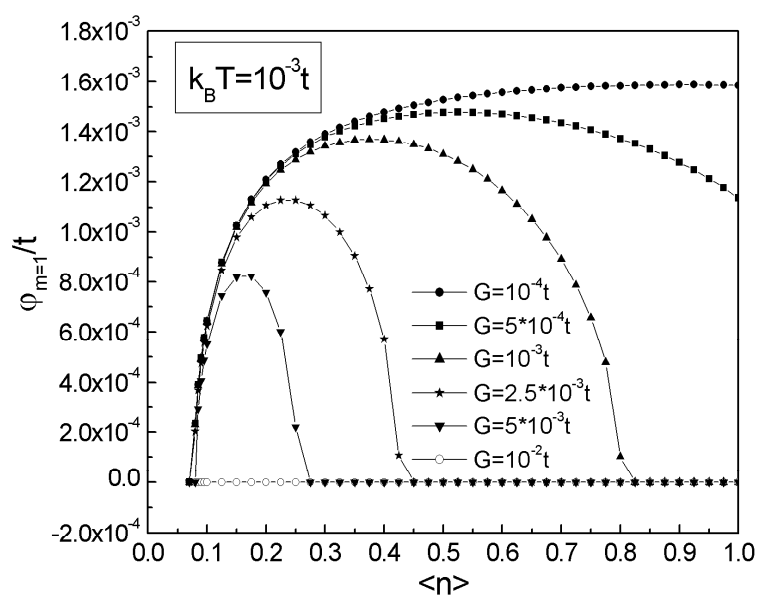

Fig. 4 Dependence of the function $\varphi_{m=1}$ on the average number of electrons at the node $\langle n\rangle$ for selected values of the $G$ parameter; $\mathrm{k}_{\mathrm{B}} T=10^{-3} t$ was assumed.
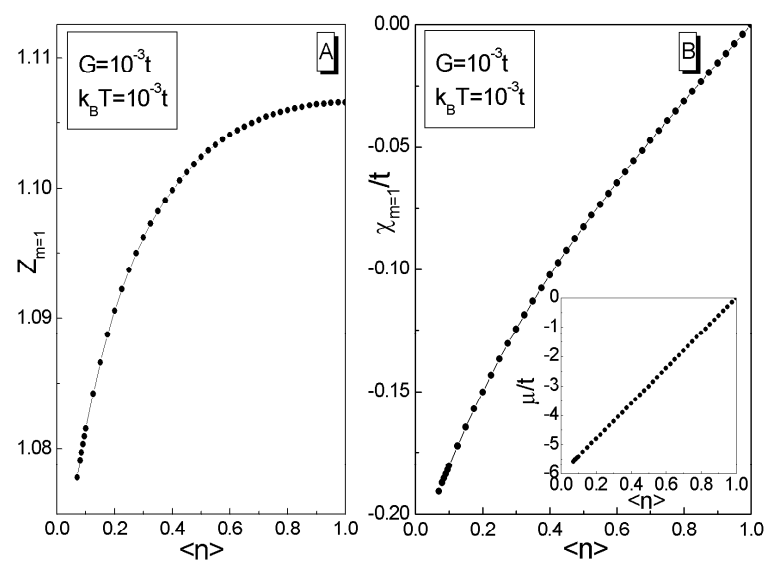

Fig. 5 (A), (B) Dependence of the functions $Z_{\mathrm{m}=1}$ and $\chi_{m=1}$ on the average number of electrons at the node. The insert in (B) shows the chemical potential as a function of $\langle n\rangle ; G=$ $10^{-3} t$ and $\mathrm{k}_{\mathrm{B}} T=10^{-3} t$ were assumed.

The open form of the $\Delta(T)$ function near the critical temperature is shown in Figs. $7 \mathrm{a}$ and $7 \mathrm{~b}$. The dependence of the order parameter presented in these figures can be described by the simple formula:

$\Delta(T)=\Delta\left(T_{1}\right) \sqrt{1-\left(\frac{T-T_{1}}{T_{c}-T_{1}}\right)^{\alpha}}$

where, for both cases, $\mathrm{k}_{\mathrm{B}} T_{1}=10^{-3} t$; for Fig. 7a: $\Delta\left(T_{1}\right)=143 \times 10^{-5} t$ and $\alpha=1.0746$; for Fig. 7b: $\Delta\left(T_{1}\right)=$ $756 \times 10^{-6} t$ and $\alpha=1.0193$.
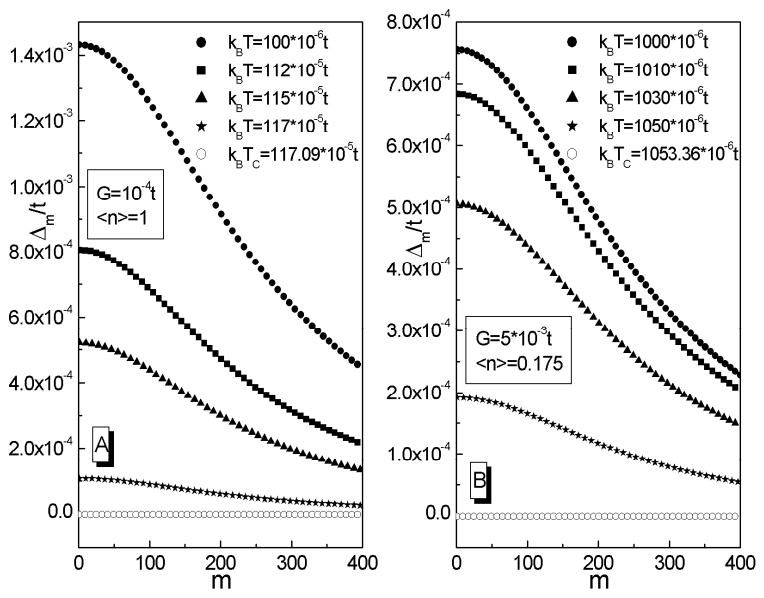

Fig. 6 Dependence of the function $\Delta_{m}$ on the Matsubara frequency for selected values of temperature; (A) $G=10^{-4} t$ and $\langle n\rangle=1$ were assumed, (B) $G=5 \times 10^{-3} t$ and $\langle n\rangle=0.175$ were assumed.

\section{Summary}

A model that describes the properties of a superconducting phase in the presence of a pseudogap has been proposed and carefully analyzed in the present paper. It is assumed that the superconducting state is induced by electron-phonon interaction, but the pseudogap is related to the presence of local charge density waves in the system.

As a result of the analysis, Eliashberg equations including the pseudogap have been derived and then solved numerically.

The most relevant result obtained here is directly connected with the determined phase diagram (see Fig. 4). It has been shown that the obtained diagram qualitatively reproduces the form of the phase diagram for the superconducting phase in $\mathrm{BaPb}_{1-x} \mathrm{Bi}_{x} \mathrm{O}_{3}$. It has to be pointed out that in the future, the analyzed model should enable a quantitative description of the superconducting state in BPBO. In order to achieve this, the exact form of the Eliashberg function for BPBO should be determined and the maximum width of the pseudogap $(G)$ should be defined on the basis of the experimental results. A qualitative analysis of this type can be performed with the possibilities of modern computer equipment and has been successfully conducted for such compounds as $\mathrm{Al}, \mathrm{Pb}$ or $\mathrm{MgB}_{2}$ [26].

A more precise description of the obtained results is presented below. First of all, the pseudogap very strongly reduces the order parameter of the superconducting phase, $\Delta$. The $\Delta$ parameter decreases with increasing width and depth of the pseudogap, which is directly connected to the lowering of the number of available electronic states near the Fermi surface. It is worth mentioning that near an appropriately large $G$ value, the superconducting 

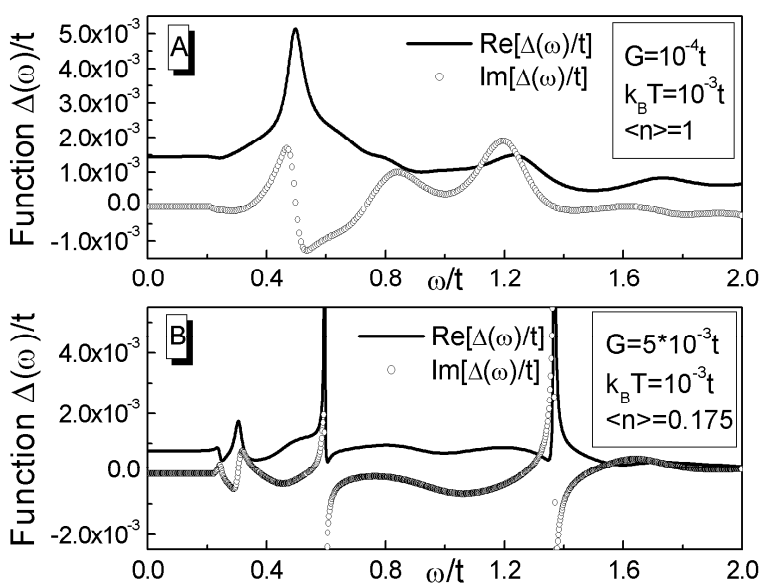

Fig. 7 The order parameter as a function of the temperature near $T_{\mathrm{c}}$; (A) $G=10^{-4} t$ and $\langle n\rangle=1$ were assumed, (B) $G=5 \times 10^{-3} t$ and $\langle n\rangle=0.175$ were assumed.

phase begins to vanish from the side of the high values of the $\langle n\rangle$ parameter. The dependence of the order parameter on the temperature near the critical temperature, in the case of a small and a large pseudogap, was also analyzed in the present paper. It was shown that with increasing size of the pseudogap the analytical form of the $\Delta(T)$ function changes; $\alpha=1.0746$ for a small pseudogap and $\alpha=1.0193$ for a large one.

Based on the presented results it has been proven that the pseudogap does not have any effect on the values of the remaining solutions of the Eliashberg equations e.g. the wave function renormalization factor and energy shift function.

\section{Appendix A}

The chemical potential of the electron gas was calculated with the assumption that the electron effective mass is equal to the bare electron mass $\left(Z_{m}=1\right)$, the many body effects do not renormalize the value of the chemical potential $\left(\chi_{m}=0\right)$ and the system is in the normal state $\left(\varphi_{m}=0\right)$. The equation that describes the chemical potential can be brought into an algebraic form, which facilitates the numerical analysis significantly. Details of the procedure are given below:

$$
\langle n\rangle=1-\frac{2}{\beta} \sum_{\mathrm{k}} \sum_{m=-\infty}^{+\infty}\left[1-\frac{G^{2}\langle n\rangle^{3}}{\omega_{m}^{2}+[G\langle n\rangle]^{2}}\right] \frac{\varepsilon_{\mathrm{k}}-\mu}{\omega_{m}^{2}+\left(\varepsilon_{\mathrm{k}}-\mu\right)} \approx 1-\mathrm{H}(\langle n\rangle, G, \mu)
$$

The $\mathrm{H}(\langle n\rangle, G, \mu)$ function is defined by the expression:

$$
\mathrm{H}(\langle n\rangle, G, \mu) \equiv \rho(0)\left[I_{1}+G\langle n\rangle^{2} \operatorname{Tanh}\left[\frac{\beta G\langle n\rangle}{2}\right] I_{2}-G^{2}\langle n\rangle^{3} I_{3}\right]
$$

where

$$
\begin{aligned}
& I_{1} \equiv \int_{-W}^{W} \operatorname{Tanh} \frac{\beta(\varepsilon-\mu)}{2} d \varepsilon=\frac{2}{\beta} \ln \left[\frac{\operatorname{Cosh}\left[\frac{\beta(W-\mu)}{2}\right]}{\operatorname{Cosh}\left[\frac{\beta(W+\mu)}{2}\right]}\right] \\
& I_{2} \equiv \int_{-W}^{W} \frac{\varepsilon-\mu}{(\varepsilon-\mu)^{2}-[G\langle n\rangle]^{2}} d \varepsilon=\frac{1}{2} \ln \left[\frac{[G\langle n\rangle]^{2}-(W-\mu)^{2}}{[G\langle n\rangle]^{2}-(W+\mu)^{2}}\right] \\
& I_{3} \equiv \int_{-W}^{W} \frac{1}{(\varepsilon-\mu)^{2}-[G\langle n\rangle]^{2}} \operatorname{Tanh} \frac{\beta(\varepsilon-\mu)}{2} d \varepsilon \\
& =-\frac{2}{\beta G\langle n\rangle}\left[\operatorname{arcTanh}\left(\frac{\mathrm{W}-\mu}{\mathrm{G}\langle\mathrm{n}\rangle}\right) \operatorname{Tanh}\left[\frac{\beta(\mathrm{W}-\mu)}{2}\right]-\operatorname{arcTanh}\left(\frac{\mathrm{W}+\mu}{\mathrm{G}\langle\mathrm{n}\rangle}\right) \operatorname{Tanh}\left[\frac{\beta(\mathrm{W}+\mu)}{2}\right]-\operatorname{Sisi}\right]
\end{aligned}
$$

The symbol Sisi denotes a finite integral that does not have a primitive function; the formula that defines the Sisi integral and its value is given below:

Sisi $\equiv \int_{-\frac{\beta(\mathrm{W}+\mu)}{2}}^{\frac{\beta(\mathrm{W}-\mu)}{2}} \operatorname{arcTanh}\left[\frac{2 \mathrm{x}}{\beta \mathrm{G}\langle\mathrm{n}\rangle}\right] \operatorname{Sech}^{2}(\mathrm{x}) \mathrm{dx} \approx \int_{-\infty}^{+\infty} \operatorname{arcTanh}\left[\frac{2 \mathrm{x}}{\beta \mathrm{G}\langle\mathrm{n}\rangle}\right] \operatorname{Sech}^{2}(\mathrm{x}) \mathrm{dx}=0$ 
The limits of the integral in Eq. A6 were shifted to $\pm \infty$ because of the values of the considered physical quantities and the form of the integrated function.

\section{Acknowledgements}

The authors would like to thank Prof. K. Dziliński, Prof. Z. Bąk and Prof. A. Khater for creating excellent working conditions.

\section{References}

[1] A.W. Sleight, J.L. Gillson, P.E. Biertedt, Solid State Commun. 17 (1975) 27.

[2] H. Fröhlich, Phys. Rev. 79 (1950) 845.

[3] H. Fröhlich, Proc. Roy. Soc. A 223 (1954) 296.

[4] Y. Nambu, Phys. Rev. 117 (1960) 648.

[5] G.M. Eliashberg, Soviet. Phys. JETP 11 (1960) 696.

[6] P.B. Allen, B. Mitrovi'c, In: H. Ehrenreich, F. Seitz, D. Turnbull (Eds.), Solid State Physics: Advances in Research and Applications, Academic Press, New York, 1982, Vol. 37, p. 1.

[7] F. Marsiglio, J.P. Carbotte, Electron-Phonon Superconductivity, In: K.H. Bennemann, J.B. Ketterson (Eds.), Handbook on Superconductivity: Conventional and Unconventional Superconductors, Springer, Berlin, 2003.

[8] O. Navarro, Physica C 265 (1996) 73.

[9] R. Szczęśniak, Acta Phys. Pol., A 103 (2006) 179.

[10] J.B. Boyce, F. Bridges, T. Claeson, Phys. Scr., T 42 (1992) 71.

[11] D.E. Cox and A.W. Sleight, Acta Crystallogr. B 35 (1979) 1.
[12] C. Chaillout, A. Santoro, J.P. Remeika, A.S. Cooper, G.P. Espinosa, N. Marezio, Solid State Commun. 65 (1988) 1363.

[13] S. Pei, N.J. Zaluzec, J.D. Jorgensen, B. Dabrowski, D.G. Hinks, A.W. Mitchell, D.R. Richards, Phys. Rev. B 39 (1989) 811.

[14] L.F. Mattheiss, D.R. Hamann, Phys. Rev. B 28 (1983) 4227.

[15] J.B. Boyce, F.G. Bridges, T. Claeson, T.H. Geballe, J.M. Remeika, Phys. Rev. B 41 (1990) 6306.

[16] J.B. Boyce, F.G. Bridges, T. Claeson, T.H. Geballe, G.G. Li, A.W. Sleight, Phys. Rev. B 44 (1991) 6961.

[17] S. Tajima, S. Ushida, A. Masaki, H. Takagi, K. Kitazawa, S. Tanaka, A. Katsui, Phys. Rev. B 32 (1985) 6302.

[18] S. Tajima, S. Ushida, A. Masaki, H. Takagi, K. Kitazawa, S. Tanaka, S. Sugai, Phys. Rev. B 35 (1987) 696.

[19] H. Sato, S. Tajima, H. Takagi, S. Uchida, Nature 338 (1989) 241.

[20] R.E. Peierls, Quantum Theory of Solids, Oxford University Press, Oxford, 1955.

[21] H. Böttger, Principles of the Theory of Lattice Dynamics, Akademie Verlag, Berlin 1983.

[22] R. Szczęśniak, M. Mierzejewski, J. Zieliński, Physica C 355 (2001) 126.

[23] V.Z. Kresin, H. Gutfreund, W.A. Little, Solid State Commun. 51 (1984) 339.

[24] V.Z. Kresin, Phys. Lett. A 122 (1987) 434.

[25] K.S.D. Beach, R.J. Gooding, F. Marsiglio, Phys. Rev. B 61 (2000) 5147.

[26] R. Szczęśniak, Phys. Status Solidi B 244 (2007) 2538.

[27] R. Szczęśniak, Solid State Commun. 145 (2008) 137.

Proceeding of the XVI International Seminar on Physics and Chemistry of Solids,

Lviv, June 6-9, 2010. 